OPEN ACCESS

Edited by:

Guochun Yan,

Central South University, China

Reviewed by:

Yunjian Liu,

Jiangsu University, China

Feixiang Wu,

Central South University, China

Wanlin Wang,

University of Wollongong, Australia

*Correspondence:

Hailong Wang

merrick_wh/@126.com

Specialty section:

This article was submitted to

Electrochemistry,

a section of the journal

Frontiers in Chemistry

Received: 23 June 2020

Accepted: 20 July 2020

Published: 09 September 2020

Citation:

Wang T, Ren $K$, He M, Dong W Xiao W, Pan H, Yang J, Yang Y, Liu P,

Cao Z, Ma X and Wang H (2020)

Synthesis and Manipulation of

Single-Crystalline Lithium Nickel

Manganese Cobalt Oxide Cathodes: A

Review of Growth Mechanism.

Front. Chem. 8:747.

doi: 10.3389/fchem.2020.00747

\section{Synthesis and Manipulation of Single-Crystalline Lithium Nickel Manganese Cobalt Oxide Cathodes: A Review of Growth Mechanism}

\author{
Ting Wang ${ }^{1,2}$, Keliang Ren ${ }^{1}$, Miao He ${ }^{1}$, Wenhao Dong ${ }^{1}$, Wei Xiao ${ }^{3}$, Hongyu Pan ${ }^{1}$, \\ Jia Yang ${ }^{1}$, Yang Yang ${ }^{1}$, Ping Liu ${ }^{4}$, Zhijie Cao ${ }^{1}$, Xiaobo Ma ${ }^{1}$ and Hailong Wang ${ }^{1,4 *}$
}

${ }^{1}$ Advanced Energy Storage Materials \& Devices Lab, Ningxia University, Yinchuan, China, ${ }^{2}$ Ningxia Polytechnic, Yinchuan, China, ${ }^{3}$ Hubei Key Laboratory of Electrochemical Power Sources, College of Chemistry and Molecular Sciences, Wuhan University, Wuhan, China, ${ }^{4}$ Office of Frontier Technology, Ningxia Power and Energy Storage Lithium-Ion Battery Materials Engineering Technology Research Center, Zhongwei, China

Lithium nickel manganese cobalt oxide (NMC) cathodes are of great importance for the development of lithium ion batteries with high energy density. Currently, most commercially available NMC products are polycrystalline secondary particles, which are aggregated by anisotropic primary particles. Although the polycrystalline NMC particles have demonstrated large gravimetric capacity and good rate capabilities, the volumetric energy density, cycling stability as well as production adaptability are not satisfactory. Well-dispersed single-crystalline NMC is therefore proposed to be an alternative solution for further development of high-energy-density batteries. Various techniques have been explored to synthesize the single-crystalline NMC product, but the fundamental mechanisms behind these techniques are still fragmented and incoherent. In this manuscript, we start a journey from the fundamental crystal growth theory, compare the crystal growth of NMC among different techniques, and disclose the key factors governing the growth of single-crystalline NMC. We expect that the more generalized growth mechanism drawn from invaluable previous works could enhance the rational design and the synthesis of cathode materials with superior energy density.

Keywords: layered cathode, single-crystalline, growth mechanism, lithium ion batteries, particle morphology

\section{INTRODUCTION}

Lithium nickel manganese cobalt oxide (NMC) cathodes have been critical pillars of advanced lithium ion batteries at current state (Chen et al., 2019; Xu et al., 2019; Zhou et al., 2019; Kim et al., 2020; Li et al., 2020; Wang et al., 2020b; Wu et al., 2020; Zhang, 2020; Zheng et al., 2020). The particle's crystallinity and morphology can put significant influences on the energy density, cycling stability, and rate capability of the NMC cathodes in practical applications (Liu et al., 2018; Fan et al., 2020). Most commercially available NMC are polycrystalline secondary particles aggregated by numerous nanosized primary particles, which usually maintain the spherical shape from the precursors. The polycrystalline nature of the secondary particles presents many issues, including (1) low tap density $\left(<3.3 \mathrm{~g} \mathrm{~cm}^{-3}\right)$ compared with the single-crystalline $\mathrm{LiCoO}_{2}$ cathode $\left(3.9 \mathrm{~g} \mathrm{~cm}^{-3}\right)$, which leads to an inadequate volumetric energy density (Kim et al., 2018), (2) the formation of microcracks inside the secondary particle incurred by the anisotropic lattice expansion 
and shrink during charging/discharging, which gradually deteriorates the cycling stability (Kim et al., 2018; Liu et al., 2018), and (3) the porous structure of the secondary particle prevents homogeneous carbon coating on each primary particle, which results in unsatisfactory electrical contact between the active material and the current collector (Kimijima et al., 2016b). In the recent decade, the chasing of larger gravimetric capacity has pushed the nickel content of the NMC to over $60 \%$; as a tradeoff larger gravimetric capacity, these nickel-rich NMC cathodes are suffering more serious issues caused by the polycrystalline secondary particles (Dixit et al., 2017; Kim et al., 2018; Lee et al., 2018; Liang et al., 2018; Ryu et al., 2018; Fan et al., 2020; Wang et al., 2020b). The mandatory increase of the tap density to over $3.3 \mathrm{~g} \mathrm{~cm}^{-3}$ generally leads to rapid capacity fading since the polycrystalline secondary particles would crack and collapse during high-pressure electrode pressing (Kim et al., 2018). Although more $\mathrm{Li}^{+}$ions can be extracted from Ni-rich cathodes, the electrostatic repulsion between oxygen slabs increases, too, which leads to a more drastic $c / a$ ratio variation during electrochemical cycling. As a result of the anisotropic lattice volume change, the microcrack propagation is more serious in those Ni-rich NMC cathodes (Liu et al., 2018; Fan et al., 2020). Several strategies, such as concentration gradient structure and grain boundary coating, have been developed to improve the structural stability of the polycrystalline NMC cathode (Kim et al., 2018; Ouyang et al., 2018; Su et al., 2019; Lee et al., 2020; Qu et al., 2020; Zou et al., 2020); nevertheless, the demands for higher volumetric energy density and cycling stability are still unsatisfied.

Well-dispersed single-crystalline NMC particles have been widely recognized with higher volumetric energy density and superior stability. Firstly, the individual single-crystalline NMC particle holds better structural integrity than the loosely aggregated polycrystalline particle during high-pressure pressing, and therefore higher tap density can be anticipated to achieve higher volumetric energy density (Kim et al., 2018; Zhong et al., 2020). Secondly, the lattice volume expansion/contraction in the single-crystalline NMC is isotropic, which significantly lowers the risk of microcrack evolution compared with the polycrystalline NMC (Liu et al., 2018). Thirdly, the coating of conductive agent is more homogeneous on the well-dispersed single-crystalline particles, which renders better electronic conductivity (Kimijima et al., 2016b). Single-crystalline particles have a smaller surface area for $\mathrm{Li}$ insertion/extraction reactions compared with the porous polycrystalline NMC; therefore, a median size of 1-4 um is critical for the single-crystalline NMC to maintain rate capabilities similar to those achieved by conventional polycrystalline NMC (Kimijima et al., 2016a). To date, several techniques, including solid-state sintering (Huang et al., 2011; Zhu et al., 2012; Lin et al., 2015; Wang et al., 2016; Jiang et al., 2017; Fan et al., 2020; Zhong et al., 2020), rheological reaction (Han et al., 2010), and molten flux growth (Kim, 2012; Kimijima et al., 2016a,b; Jiang et al., 2017), have been used to synthesize single-crystalline NMC cathode; equivalent electrochemical performances have been achieved in terms of capacity and rate capability compared with polycrystalline NMC. Moreover, the cycling stability of single-crystalline NMC outperforms the polycrystalline product. Each work had discussed the growth mechanism, but the understandings are mostly based on individual techniques. A deep review and analysis can help draw a more fundamental and universal growth mechanism of the single-crystalline NMC, which would facilitate the knowledge-based improvement of synthesis techniques.

\section{GROWTH MECHANISM: GENERAL CONSIDERATIONS FOR NMC CATHODE}

A crystallization process generally comprises of two stages: First, the formation of three-dimensional nuclei from the supersaturated medium (or matrix), and second, the nuclei grows into a larger crystal entity (Sangwal, 2018). In the first stage, supersaturation is a prerequisite for the new phase to nucleate, which can be reached by concentration fluctuation, solvent evaporation, and cooling of the medium. As long as the nuclei's size can exceed the critical value $R$ (Mittemeijer, 2010):

$$
R=\frac{2 \gamma}{\Delta G_{\text {chem }}^{v}+\Delta G_{\text {strain }}^{v}}
$$

where $\gamma$ is the interfacial energy per unit (interfacial) area, $\Delta G_{c h e m}^{v}$ is the energy contribution driving the phase transformation, and $\Delta G_{\text {strain }}^{v}$ is the strain energy per unit volume.

The phase change-incurred free energy drop would overcome the increase of surface free energy, and the nuclei would be stable. Otherwise, it would be dissolved into the medium. In the second stage, the mass transportation controls the growth rate of crystals when the mean distance between grains is far enough (Lifshitz et al., 1961). As time passes by, the closed system tends to achieve a minimal energy state by reducing the overall surface energy, where the Ostwald ripening (grain coarsening) process would dominate (Sangwal, 2018).

The synthesis of NMC cathode usually uses a mixture of transition metal hydroxides/nitrides/sulfates and $\mathrm{LiOH} / \mathrm{Li}_{2} \mathrm{CO}_{3}$ as the precursor medium. A minimal heat input above $200^{\circ} \mathrm{C}$ can trigger enough concentration fluctuation for the nucleation of NMC (Zhu et al., 2012); however, the grain growth is negligible until the calcination temperature reaches the melting point of $\mathrm{LiOH} / \mathrm{Li}_{2} \mathrm{CO}_{3}$. In the melts of $\mathrm{Li}$ precursors, the mass transportation is accelerated, and so is the crystal growth (Grigorova et al., 2011). When the calcination temperature reaches above $800^{\circ} \mathrm{C}$ (Zhu et al., 2012; Kong et al., 2019), the slight evaporation of $\mathrm{Li}_{2} \mathrm{O}$ can further facilitate the mass transport and results in significantly accelerated crystal growth since the homogeneous distribution of transition metal ions has usually been achieved by coprecipitation or milling, and the NMC lattice formation mostly relies on oxygen and lithium migration, whereas the phase and the composition of NMC would begin to change when calcined at 900 $1,000^{\circ} \mathrm{C}$ due to vigorous lithium volatilization (Wang et al., 2017; Zhao et al., 2017). To date, many works have been devoted to synthesize single-crystalline NMC cathode and various methods have been developed, which can be generally 
categorized into solid-state reaction, solid-liquid reaction, and molten flux growth. As illustrated in Figure 1, the distinct differences in nucleation, mass transportation, and growth environment yield differences in agglomeration, size, and shape of NMC product. In the following sections, we will review and discuss the mechanisms and the key factors dominating the growth of single-crystalline NMC cathode in different methods.

\section{Solid-State Reaction}

The most prominent feature of solid-state reaction is that the NMC product can easily inherit the morphology from the solid precursors (Figure 1A). The nucleation of the new phase takes place in the solid precursor medium, and the growth of grains is constrained by the mass transport inside the rigid lattice. As a consequence, most commercial NMC products synthesized through the coprecipitation route inherit the agglomerated polycrystalline spheres from the $\left(\mathrm{Ni}_{x} \mathrm{Mn}_{y} \mathrm{Co}_{z}\right)(\mathrm{OH})_{2}$ precipitates. Constrained by the solid precursor medium, it is very hard to obtain well-dispersed single-crystalline NMC particles in single-time calcination. Even under extremely high temperatures over $1,000^{\circ} \mathrm{C}$, the greatly enhanced mass transportation between grain boundaries can only yield 500-nm primary particles closely aggregated together (Zhu et al., 2012). Li et al. (2017), Zhong et al. (2020), and Fan et al. (2020) have demonstrated that additional grinding and multiple calcinations can help obtain 1-3-um singlecrystalline NMC below $1,000^{\circ} \mathrm{C}$ (Figure 1B). During heating or calcination, additional grinding can break the agglomeration and expose a large number of fresh surfaces, where the grain boundary diffusion can be greatly enhanced and the energy barrier for crystal growth can be overcome easier. Altering the agglomeration state of the solid precursor medium and elevating the calcination temperature can alleviate agglomeration and improve the crystallinity. Huang et al. (2011) firstly prepared highly porous solid precursors through the violent evaporation of volatile species from a gel, then solid-state calcination between 900 and $950^{\circ} \mathrm{C}$ produces $500-\mathrm{nm}$ single-crystalline NMC111 particles loosely interconnected with each other. Lin et al. (2015) added $\mathrm{H}_{2} \mathrm{O}_{2}$ during the coprecipitation process, which then decompose and produce a large amount of $\mathrm{O}_{2}$ gas bubbles to disperse the precursors, and finally they obtained loosely connected 300-nm NMC111 single crystals after $800^{\circ} \mathrm{C}$ calcination. Jiang et al. (2017) used the eutectic mixture of $\mathrm{LiOH}$ and $\mathrm{LiNO}_{3}$ as an alternative $\mathrm{Li}$ source for solid-state reaction, and the melting point of the eutectic mixture was below $200^{\circ} \mathrm{C}$, which is much lower than that of the conventional $\mathrm{LiOH}$ precursor $\left(500^{\circ} \mathrm{C}\right)$. As a result of the sufficient and fast lithium diffusion, they successfully synthesized NMC111 particles of about $700 \mathrm{~nm}$ after $900^{\circ} \mathrm{C}$ calcination, and the aggregation is alleviated. In addition to the coprecipitation method, Wang et al. (2016) firstly prepared a well-dispersed $\mathrm{M}\left(\mathrm{C}_{2} \mathrm{O}_{4}\right) \cdot 2 \mathrm{H}_{2} \mathrm{O}(\mathrm{M}=\mathrm{Ni}, \mathrm{Mn}$, and $\mathrm{Co})$ mixture through hydrothermal reaction and then ground them with $\mathrm{LiNO}_{3}$. Finally, they obtained NMC622 single crystals of about $1-2 \mathrm{um}$, and the aggregation is very slight after $800-900^{\circ} \mathrm{C}$ calcinations (Figure 1C).

\section{Solid-Liquid Rheological Reaction}

The growth of a single crystal is easier in solid-liquid rheological reaction than in solid-state calcination, which has been successfully used to synthesize single-crystal $\mathrm{Ni}(\mathrm{Sal}) 2 \cdot 4 \mathrm{H}_{2} \mathrm{O}[\mathrm{Sal}$ $=\mathrm{C}_{6} \mathrm{H}_{4} \mathrm{OH}(\mathrm{CO})_{2}$ ] (Wang et al., 2003) and $\mathrm{Cu}\left(\mathrm{C}_{6} \mathrm{H}_{5} \mathrm{CO}_{2}\right) 3 \mathrm{H}$ (Zhao et al., 2011). The rheological body is a uniform mixture of solid precursors and liquid substances (Tang et al., 2002), in which mass transportation is certainly easier than in pure solid state and phase nucleation could be either in the solid phase or in the supersaturated region of the liquid phase (Figure 1D). Maintaining the rheological body during calcination is critical to obtain the single-crystalline NMC particles. Unfortunately, most liquid substances used in rheological reactions cannot exist at temperatures higher than $300^{\circ} \mathrm{C}$; the rhetorical body in the mixing or preheating process can only improve the element distribution, but the final product still inherits the polycrystalline and the agglomeration nature of the solid precursors (Xiao et al., 2004; Li et al., 2008, 2014, 2016; Shi et al., 2009, 2014). Han et al. used the conventional rheological reaction to uniformly insert the very fine nanoscale $\mathrm{NiO}$ and $\mathrm{Co}_{3} \mathrm{O}_{4}$ into spherical amorphous $\mathrm{MnO}_{2}$ at the first step; interestingly, during $950^{\circ} \mathrm{C}$ calcination, the assembled nanostructured precursor easily melted and formed a glass-like rheological body, as displayed in Figures 1E,F. Eventually, well-dispersed singlecrystalline NMC111 particles of about 2-4 um were successfully obtained (Han et al., 2010).

\section{Crystal Growth From Molten Salt Flux}

Crystal growth in molten salt flux has several advantages, including (1) facilely obtaining multicomponent inorganic crystals at temperatures far below the melting point of the precursor solute, (2) crystal growth is free of the rigid mechanical constraint of the solid precursors, and (3) the salts are generally cheap and environmentally friendly. As illustrated in Figure 1G, firstly, the precursor solutes are dissolved in the molten salts and then reach supersaturation, through either flux evaporation or cooling, to enable nucleation. After nucleation, the crystal growth in the molten flux is subject to mass transportation and the Ostwald ripening process. The holding temperature, solute concentration, and reaction time are key factors that influence the single-crystalline NMC products in the flux growth method (Wang et al., 2020a).

First of all, the holding temperature should be high enough to melt the salt and enable the precursor solute to dissolve into the flux. Kim (2012) tried to dissolve the coprecipitated $\left(\mathrm{Ni}_{0.8} \mathrm{Mn}_{0.1} \mathrm{Co}_{0.1}\right)(\mathrm{OH})_{2}$ into $\mathrm{NaCl}$ (melting point, $800^{\circ} \mathrm{C}$ ) and $\mathrm{KCl}$ (melting point, $770^{\circ} \mathrm{C}$ ) at $800^{\circ} \mathrm{C}$. The fully melted $\mathrm{KCl}$ salt yields well-separated single-crystalline NMC811 particles, while the partially melted $\mathrm{NaCl}$ salts generated $\mathrm{NMC} 811$ particles that inherit the agglomeration nature of the $\left(\mathrm{Ni}_{0.8} \mathrm{Mn}_{0.1} \mathrm{Co}_{0.1}\right)$ $(\mathrm{OH})_{2}$ precursor. Generally, the higher holding temperature can give rise to a lower viscosity of the melt; both the solubility and the mobility of solute components can be improved by a higher holding temperature, which will result in a larger size of the single-crystalline particles (Teshima et al., 2011; Kim, 2012; Kimijima et al., 2016b). It is worth to note that the gravimetric capacity of the single-crystalline NMC could 


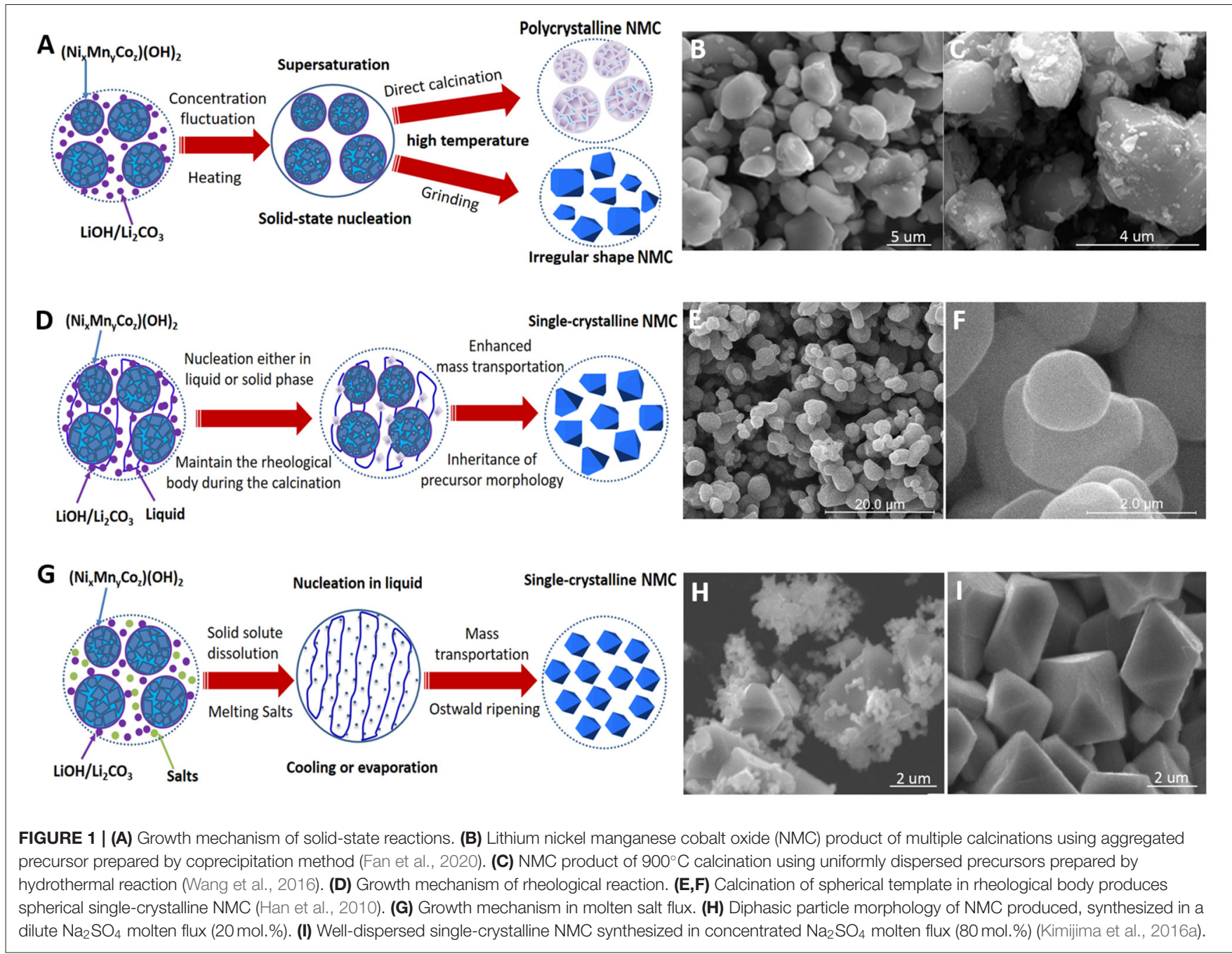

be seriously dampened when the holding temperature exceeds $900^{\circ} \mathrm{C}$ (Kim, 2012; Kimijima et al., 2016b) since a large amount of $\mathrm{Li}$ is volatilized.

In a flux with very low solute concentration, where all solutes are fully dissolved, supersaturation and nucleation are almost randomly achieved, and the subsequent crystal growth is independent with each other. Under such condition, a diphasic particle morphology (Figure 1H) of the final product is commonly seen, in which the micron-sized crystals with well-developed facets coexist with nanoscale crystals possessing irregular shapes (Teshima et al., 2011; Kimijima et al., 2016a). When the solute is partially dissolved, the remaining solid solute would play as sources for supplying reactive mass into the salt flux by diffusion. Supersaturation is easier to reach in the flux region adjacent to the undissolved solute to enable nucleation. In such a scenario, the solute concentration of the mixture can be considered as the average distance among these undissolved solid solute particles. Increasing the solute concentration would decrease the average distance among the sources for supplying mass, which would facilitate a fast and sufficient transportation of reactive mass to the growing crystals.
As a result, most flux growth works have reported that the particle size of single-crystalline NMC increases with increasing solute concentration (Kim, 2012; Kimijima et al., 2016a,b), as compared in Figures 1H,I.

The crystal growth in the molten flux inevitably introduces the additional solid/liquid interfacial energy, which varies with the interface area. Thermodynamically, the system tends to reach a more stable state by decreasing the interfacial energy, and this could be achieved by increasing the average size of the crystals (Ostwald ripening) (Sangwal, 2018). With increasing reaction time, the smaller crystals would dissolve into the flux to feed the larger crystals and make them grow larger, and eventually the crystal size distribution can be improved (Kimijima et al., 2016a,b).

\section{MANIPULATION OF MORPHOLOGY}

Manipulating the shape and the crystal orientation of cathode particles could help gain advantages in both tap density 
and electrochemical performances. As described above, the nucleation and the growth of single-crystalline NMC generally take place in a less-constrained environment, where several measures can be adopted in different stages to manipulate the morphology.

\section{Control of Shape}

In the growth process which is closely associated with solid precursors, the rigid solid structure and the stagnant mass transportation make it hard for the final product to deviate from the shape of the precursors (Huang et al., 2011; Wang et al., 2016; Zhong et al., 2020). On the other hand, the shape inheritance from solid precursors can be utilized as templates for the final products' shape control. Han et al. (2010) have used the spherical amorphous $\mathrm{MnO}_{2}$ particles as growth templates in a rheological reaction to synthesize single-crystalline NMC111. In the glass-like mixture $\left(\mathrm{LiOH}, \mathrm{NiO}\right.$, and $\left.\mathrm{Co}_{3} \mathrm{O}_{4}\right)$ of oxide melt and amorphous $\mathrm{MnO}_{2}$, the mass transportation and the ionic rearrangement are constrained by the solid sphere of $\mathrm{MnO}_{2}$, of which the spherical shape also helps minimize the surface energy during growth. Eventually, spherical single-crystalline NMC111 particles were successfully synthesized. Jiang et al. (2017) firstly synthesized polygon $\left(\mathrm{Ni}_{0.29} \mathrm{Mn}_{0.33} \mathrm{Co}_{0.38}\right)(\mathrm{OH})_{2}$ nanoplates with only $20-\mathrm{nm}$ thickness and then calcined in a $\mathrm{LiOH}-\mathrm{LiNO}_{3}$ mixture melt at $900^{\circ} \mathrm{C}$. During the solid-liquid reaction, lithium and oxygen in the melt can easily attach and react on the surfaces of the nanoplates; by rapid diffusion of lithium and oxygen, the melt glue and assemble these polygon nanoplates along their axial direction, and eventually, polygon-shaped single-crystalline NMC with thickness of 200-500 nm have been obtained.

In the case of growth in liquid medium such as molten salt flux, the surface atoms of the infant nucleus are in high ratio and loosely bonded, which are easily influenced by subtle mechanical or thermal perturbation in the medium. The initial morphologies of the infant nucleus are random and less constrained by their lattice. If the NMC nucleus grows unimpeded, the idiomorphic growth of crystal ultimately acquires a well-defined octahedral shape, and this is achieved by sufficient mass transportation from the supersaturated medium and efficient mass migration on the crystal surfaces.

Takeshi et al. (Kimijima et al., 2016a) reported that singlecrystalline NMC111 particles grown in molten $\mathrm{Na}_{2} \mathrm{SO}_{4}$ flux exhibit an octahedral shape with a flat surface, but which, when grown in the $\mathrm{Li}_{2} \mathrm{SO}_{4}$ flux, exhibit a blurred shape and a smaller size. The difference can be ascribed to the higher solubility of the $\mathrm{Li}_{2} \mathrm{SO}_{4}$ flux, which decreases the supersaturation degree of the medium and cannot drive sufficient precipitation from the flux to attach on the crystals' surfaces. Single-crystalline NMC811 particles grown in $\mathrm{KCl}$ exhibit an isotropic shape, whereas the octahedral shape with well-developed (003) and (11-1) facets is acquired in the $\mathrm{NaCl}$ flux. Kim proposed that the $\mathrm{KCl}$ flux may reduce the surface energy of the facet (Kim, 2012), but the exact influence of the $\mathrm{KCl}$ and the $\mathrm{NaCl}$ fluxes on the surface energy of the NMC crystal has yet to be clarified. We have noticed that the $\mathrm{KCl}$ flux can dissolve the solute precursor easier than the $\mathrm{NaCl}$ flux in the work of Kim (2012), which implies that the solubility of the $\mathrm{KCl}$ flux is higher than that of the $\mathrm{NaCl}$ flux. Moreover, the NMC811 particles $(<2 \mu \mathrm{m})$ grown in the $\mathrm{KCl}$ flux are much smaller than those $(>5 \mu \mathrm{m})$ in the $\mathrm{NaCl}$ flux. All of these imply a less-sufficient mass transportation in the $\mathrm{KCl}$ flux due to higher solubility and less supersaturation.

It can be concluded that the morphology of singlecrystalline NMC products is mostly dominated by their growth environment. The solid-state phase transition is rigidly constrained by a hard template, and the morphology inheritance from the precursor is inevitable unless extremely high temperatures significantly break the rigid constraint of solids. The growth in liquid or liquid/solid mixture is freer of mechanical constraint. Therefore, the growth of well-defined crystal planes would be easier. Without the rigid constraint of the solid precursor, manipulation of the morphology is feasible by altering the chemical or the thermal conditions. Whatever the growth environment is, the particle size of the final product is mostly dominated by the mass transportation rate in a limited growth time. Generally, a fast mass transportation, which is enabled by either a high temperature or a steep concentration gradient or a dramatic variation of solubility, can facilitate crystal growth or grains to coalesce to yield large primary particles.

\section{Control of Facet}

Owing to the hexagonal layered structure, the NMC crystal can enable fast lithium transportation through the $\{010\}$ facets while impeding lithium migration through the $\{001\}$ facets. Obviously, the highly exposed $\{010\}$ facets of the NMC cathode would give rise to enhanced rate capabilities. Unfortunately, the surface energy of the $\{010\}$ facets is higher than that of the $\{001\}$ facets, which leads to a higher growth rate and eventually the disappearance of the $\{010\}$ facets in an uncontrolled growth process. In the recent decade, many works have successfully synthesized NMC products with highly exposed $\{010\}$ facets through using a surface capping agent, adjusting the $\mathrm{pH}$ value, and modifying the tank reactor (Wei et al., 2010; Fu et al., 2013; Chen et al., 2014; Hua et al., 2017; Yu et al., 2017; Ju et al., 2018; Su et al., 2018; Zhou et al., 2018; Dong et al., 2019; Xiang et al., 2019). Dramatically increasing the $\{010\}$ facets generally causes a serious agglomeration, owing to the large surface area. Although a porous or a hierarchical structure can help expose the $\{010\}$ facets of the agglomerated secondary particles, it may further decrease the tap density of the NMC product. The synthesis of well-dispersed single-crystalline NMC with preferred facets still remains a great challenge. There are some works that can give clues for tuning the facets of single-crystalline NMC. Fu et al. (2013) added a large amount of polyvinylpyrrolidone during the coprecipitation process, creating a viscous environment which dramatically slows the nucleation rate. The limited number of nuclei adsorbed by PVP grows into nanoplates in the length of $200 \mathrm{~nm}$ in the solution. They further tuned the calcination temperature to increase the thickness, and eventually, singlecrystalline NMC111 with exposed $\{010\}$ facets are obtained. Kim (2012) and Takeshi et al. (Kimijima et al., 2016a) all have pointed out that the $\mathrm{Na}^{+}$ions in the molten salt flux can help the NMC crystallites expose more $\{003\},\{101\}$, and $\{111\}$ facets. Since $\mathrm{Na}^{+}$ ions were not found to incorporate into the NMC lattice, it was 
believed that the surface energy or the surface mass migration conditions can be influenced by $\mathrm{Na}^{+}$ions in flux.

\section{DISCUSSION AND PERSPECTIVE}

As discussed above, the main purpose of developing a singlecrystalline NMC cathode is to achieve superior volumetric energy density and cycling stability. During the development progress, a median size of 1-4 um is found critical for a single-crystalline NMC cathode to maintain competitive rate capabilities. Solidstate reaction with additional grinding and calcination can facilely produce single-crystalline NMC, but the particles' shape is irregular and mild agglomeration still remains. Molten-fluxbased methods have exhibited superiority in the synthesis of welldispersed single-crystalline NMC cathode, including flexibility of the precursor's choice and easy to acquire micron-metersized well-crystallized primary particles. In addition, the particle size distribution is tunable in the Ostwald ripening process by altering the reaction temperature and the holding time, whereas the manipulation of crystal shape and the preferred facets is still premature in molten-flux-based growth. The rheological reaction exhibits a promising synergic effect of rapid mass transportation in liquid and morphology inheritance from solid precursors. However, the speculation on the details of the solid-liquid reaction process still lacks complete physical chemistry insights.

In view of the capital cost for industrial production, the solidstate synthetic route requires almost no facility investment to produce single-crystalline NMC. Grinding, multiple calcinations, and a well-dispersed precursor can be realized in the current facilities. However, both the rheological reaction and the molten flux growth route require modifications on the conventional tunnel furnace to prevent an explosive spillover of hot liquid and to more accurately control the cooling rate. In view of energy consumption, the molten flux growth can yield micronsized single-crystalline NMC in one-step calcination below $900^{\circ} \mathrm{C}$, whereas the solid-state synthetic route generally requires multiple steps, with a final heating above $900^{\circ} \mathrm{C}$. In view of

\section{REFERENCES}

Chen, L., Su, Y., Chen, S., Li, N., Bao, L., Li, W., et al. (2014). Hierarchical Li1.2Ni0.2Mn0.6O2 nanoplates with exposed $\{010\}$ planes as high-performance cathode material for lithium-ion batteries. Adv. Mater. 26, 6756-6760. doi: 10.1002/adma.201402541

Chen, Z., Gong, X., Zhu, H., Cao, K., Liu, Q., Liu, J., et al. (2019). High performance and structural stability of $\mathrm{K}$ and $\mathrm{Cl} \mathrm{Co}-$ Doped $\mathrm{LiNi}_{0.5} \mathrm{Co}_{0.2} \mathrm{Mn}_{0.3} \mathrm{O}_{2}$ cathode materials in 4.6 voltage. Front. Chem. 6:643. doi: 10.3389/fchem.2018.00643

Dixit, M., Markovsky, B., Schipper, F., Aurbach, D., and Major, D. T. (2017). Origin of structural degradation during cycling and low thermal stability of nirich layered transition metal-based electrode materials. J.Phys. Chem. C 121, 22628-22636. doi: 10.1021/acs.jpcc.7b06122

Dong, H., Li, S., Liu, H., Mei, J., Liu, H., and Liu, G. (2019). Facile synthesis and electrochemical properties of $\mathrm{LiNi}_{0.8} \mathrm{Co}_{0.15} \mathrm{Al}_{0.05} \mathrm{O}_{2}$ with enlarged exposed active planes for Li-ion batteries. Ionics 25, 827-834. doi: 10.1007/s11581-018-2620-5

Fan, X., Hu, G., Zhang, B., Ou, X., Zhang, J., Zhao, W., et al. (2020). Crack-free single-crystalline Ni-rich layered NCM cathode enable superior product quality, both the molten flux growth and the rheological reaction are fascinating since the fast mass transportation and the less-constrained growth environment can produce welldispersed particles with uniform size and morphology. In addition, low-temperature calcination $\left(<900^{\circ} \mathrm{C}\right)$ can prevent significant Li/O loss.

The fully controllable synthesis of single-crystalline NMC is dependent on deep understandings of phase nucleation, mass transportation, and surface energy variations. In the past, the closed holder containing the liquid/solid reactive materials at high temperatures has hidden the details of the growth process and left puzzles of morphology variation. The recent advances on in situ transmission electron microscopy and neutron diffraction methods can help interrogate the key factors that influence single-crystalline NMC growth (Shoemaker et al., 2014; Vasquez et al., 2019; Wang et al., 2020a), and theoretical computations of surface energies and their variations in different environments would guide an efficient manipulation of crystal morphology.

\section{AUTHOR CONTRIBUTIONS}

TW searched the relevant literatures, draw the figure draft, and acquired rights/permissions for SEM pictures. HW proposed the topic, guided the analysis, and wrote the whole manuscript. All authors participated in the analysis on previous work and discussed the growth mechanisms.

\section{FUNDING}

This work was supported by Science Research Project of Ningxia Higher Institutions (NGY2018-199), National Science Foundation of China (NSFC-51462029 and 11762018), Ningxia Natural Science Foundation Project (2020AAC03258), Key Research and Development Project of Ningxia province (2018BEE03004), and Technology Leading Talent Program of Ningxia province (KJT2016003). cycling performance of lithium-ion batteries. Nano Energy 70:104450. doi: 10.1016/j.nanoen.2020.104450

Fu, F., Xu, L.-G., Wang, Q., Deng, P.-Y., Li, X., Li, T.-J., et al. (2013). Synthesis of single crystalline hexagonal nanobricks of $\mathrm{LiNi}_{1 / 3} \mathrm{Co}_{1 / 3} \mathrm{Mn}_{1 / 3} \mathrm{O}_{2}$ with high percentage of exposed $\{010\}$ active facets as high rate performance cathode material for lithium-ion battery. J. Mater. Chem. A 1, 3860-3864. doi: $10.1039 / \mathrm{c} 3$ ta01618h

Grigorova, E., Mandzhukova, T. S., Khristov, M., Yoncheva, M., Stoyanova, R., Zhecheva, E., et al. (2011). Soft mechanochemically assisted synthesis of nano-sized $\mathrm{LiCoO}_{2}$ with a layered structure. J. Mater. Sci. 46, 7106-7113. doi: 10.1007/s10853-011-5407-x

Han, X., Meng, Q., Sun, T., and Sun, J. (2010). Preparation and electrochemical characterization of single-crystalline spherical $\mathrm{LiNi}_{1 / 3} \mathrm{Co}_{1 / 3} \mathrm{Mn}_{1 / 3} \mathrm{O}_{2}$ powders cathode material for Li-ion batteries. J. Power Sources 195, 3047-3052. doi: 10.1016/j.jpowsour.2009.11.007

Hua, W., Wu, Z., Chen, M., Knapp, M., Guo, X., Indris, S., et al. (2017). Shapecontrolled synthesis of hierarchically layered lithium transition-metal oxide cathode materials by shear exfoliation in continuous stirred-tank reactors. J. Mater. Chem. A 5, 25391-25400. doi: 10.1039/C7TA08073E 
Huang, Z. D., Liu, X., M., Oh, S., W., Zhang, B., et al. (2011). Microscopically porous, interconnected single crystal $\mathrm{LiNi}_{1 / 3} \mathrm{Co}_{1 / 3} \mathrm{Mn}_{1 / 3} \mathrm{O}_{2}$ cathode material for Lithium ion batteries. J. Mater. Chem. 21, 10777-10784. doi: $10.1039 / \mathrm{cljm} 00059 \mathrm{~d}$

Jiang, X., Chu, S., Chen, Y., Zhong, Y., Liu, Y., and Shao, Z. (2017). $\mathrm{LiNi}_{0.29} \mathrm{Co}_{0.33} \mathrm{Mn}_{0.38} \mathrm{O}_{2}$ polyhedrons with reduced cation mixing as a highperformance cathode material for Li-ion batteries synthesized via a combined co-precipitation and molten salt heating technique. J. Alloy. Compd. 691, 206-214. doi: 10.1016/j.jallcom.2016.08.139

Ju, X., Huang, H., He, W., Zheng, H., Deng, P., Li, S., et al. (2018). Surfactantassisted synthesis of high energy $\{010\}$ facets beneficial to li-ion transport kinetics with layered $\mathrm{LiNi}_{0.6} \mathrm{Co}_{0.2} \mathrm{Mn}_{0.2} \mathrm{O}_{2}$. Acs Sustain. Chem. Eng. 6, 6312-6320. doi: 10.1021/acssuschemeng.8b00126

Kim, J., Cha, H., Lee, H., Oh, P., and Cho, J. (2020). Surface and interfacial chemistry in the nickel-rich cathode materials. Batter. Supercaps. 3, 309-322. doi: 10.1002/batt.201900131

Kim, J., Lee, H., Cha, H., Yoon, M., Park, M., and Cho, J. (2018). Prospect and reality of Ni-rich cathode for commercialization. Adv. Energy Mater. 8, 1702028 doi: $10.1002 /$ aenm.201702028

Kim, Y. (2012). Lithium nickel cobalt manganese oxide synthesized using alkali chloride flux: morphology and performance as a cathode material for lithium ion batteries. ACS Appl. Mater. Inter. 4, 2329-2333. doi: 10.1021/am300386j

Kimijima, T., Zettsu, N., and Teshima, K. (2016a). Growth manner of octahedralshaped $\mathrm{Li}\left(\mathrm{Ni}_{1 / 3} \mathrm{Co}_{1 / 3} \mathrm{Mn}_{1 / 3}\right) \mathrm{O}_{2}$ single crystals in molten $\mathrm{Na}_{2} \mathrm{SO}_{4}$. Crys. Growth Des. 16, 2618-2623. doi: 10.1021/acs.cgd.5b01723

Kimijima, T., Zettsu, N., Yubuta, K., Hirata, K., Kami, K., and Teshima, K. (2016b). Molybdate flux growth of idiomorphic $\mathrm{Li}\left(\mathrm{Ni}_{1 / 3} \mathrm{Co}_{1 / 3} \mathrm{Mn}_{1 / 3}\right) \mathrm{O}_{2}$ single crystals and characterization of their capabilities as cathode materials for lithium-ion batteries. J. Mater. Chem. A 4, 7289-7296. doi: 10.1039/C6TA01593J

Kong, D., Zhang, M., Xiao, Y., Hu, J., Zhao, W., Han, L., et al. (2019). Insights into the structural evolution and Li/O loss in high-Ni layered oxide cathodes. Nano Energy 59, 327-335. doi: 10.1016/j.nanoen.2019.02.059

Lee, W., Muhammad, S., Kim, T., Kim, H., Lee, E., Jeong, M., et al. (2018). New insight into Ni-rich layered structure for next-generation li rechargeable batteries. Adv. Energy Mater. 8:1701788. doi: 10.1002/aenm.201701788

Lee, W., Muhammad, S., Sergey, C., Lee, H., Yoon, J., Y.-,Kang, M., et al. (2020). Advances in the cathode materials for lithium rechargeable batteries. Angew. Chem. Int. Edit. 59, 2578-2605. doi: 10.1002/anie.201902359

Li, D., Yuan, C., Dong, J., Peng, Z., and Zhou, Y. (2008). Synthesis and electrochemical properties of $\mathrm{LiNi}_{0.85-\mathrm{x}} \mathrm{Co}_{\mathrm{x}} \mathrm{Mn}_{0.15} \mathrm{O}_{2}$ as cathode materials for lithium-ion batteries. J. Solid State Electr. 12, 323-327. doi: 10.1007/s10008-007-0394-1

Li, J., Cameron, A., R., Li, H., Glazier, S., Xiong, D., et al. (2017). Comparison of single crystal and polycrystalline $\mathrm{LiNi}_{0.5} \mathrm{Mn}_{0.3} \mathrm{Co}_{0.2} \mathrm{O}_{2}$ positive electrode materials for high voltage li-ion cells. J. Electrochem. Soc. 164, A1534-A1544. doi: 10.1149/2.0991707jes

Li, L., Feng, C., Zheng, H., He, P., and Wang, J. (2014). Synthesis and electrochemical properties of $\mathrm{LiNi}_{1 / 3} \mathrm{Co}_{1 / 3} \mathrm{Mn}_{1 / 3} \mathrm{O}_{2}$ cathode material. J. Electron. Mater. 43, 3508-3513. doi: 10.1007/s11664-014-3184-4

Li, L., Xia, L., Yang, H., Zhan, X., Chen, J., Chen, Z., et al. (2020). Solid-state synthesis of lanthanum-based oxides Co-coated $\mathrm{LiNi}_{0.5} \mathrm{Co}_{0.2} \mathrm{Mn}_{0.3} \mathrm{O}_{2}$ for advanced lithium ion batteries. J. Alloy. Compd. 832:154959. doi: 10.1016/j.jallcom.2020.154959

Li, X., Peng, H., M.-, Wang, S., Zhao, X., P.-, Huang, X., Yang, W., et al. (2016). Enhanced electrochemical performance of Zr-Modified Layered $\mathrm{LiNi}_{1 / 3} \mathrm{Co}_{1 / 3} \mathrm{Mn}_{1 / 3} \mathrm{O}_{2}$ cathode material for lithium-ion batteries. Chemelectrochem 3, 130-137. doi: 10.1002/celc.201500360

Liang, C., Longo, R., C., Kong, F., Zhang, C., Nie, Y., et al. (2018). Ab Initio study on surface segregation and anisotropy of Ni-Rich $\mathrm{LiNi}_{1-2 \mathrm{y}} \mathrm{Co}_{\mathrm{y}} \mathrm{Mn}_{\mathrm{y}} \mathrm{O}_{2}$ (NCM) $(\mathrm{y} \leq 0.1)$ cathodes. ACS Appl. Mater. Inter. 10, 6673-6680. doi: 10.1021 /acsami.7b17424

Lifshitz, I. M., and Slyozov, V., V. (1961). The kinetics of precipitation from supersaturated solid solutions. J. Phys. Chem. Solids 19, 35-50. doi: 10.1016/0022-3697(61)90054-3

Lin, C., Zhang, Y., Chen, L., Lei, Y., Ou, J., Guo, Y., et al. (2015). Hydrogen peroxide assisted synthesis of $\mathrm{LiNi}_{1 / 3} \mathrm{Co}_{1 / 3} \mathrm{Mn}_{1 / 3} \mathrm{O}_{2}$ as highperformance cathode for lithium-ion batteries. J. Power Sources 280, 263-271. doi: 10.1016/j.jpowsour.2015.01.084
Liu, G., Li, M., Wu, N., Cui, L., Huang, X., Liu, X., et al. (2018). Singlecrystalline particles: an effective way to ameliorate the intragranular cracking, thermal stability, and capacity fading of the $\mathrm{LiNi}_{0.6} \mathrm{Co}_{0.2} \mathrm{Mn}_{0.2} \mathrm{O}_{2}$ electrodes. $J$. Electrochem. Soc. 165, A3040-A3047. doi: 10.1149/2.0491813jes

Mittemeijer, E. J. (2010). Fundamentals of Materials Science: The MicrostructureProperty Relationship Using Metals as Model Systems. Berlin, Heidelberg: Springer-Verlag, 374-375

Ouyang, H., Li, X., Wang, Z., Guo, H., Peng, W., and $\mathrm{He}$, Z. (2018). Improved electrochemical performance of $\mathrm{LiNi}_{0.8} \mathrm{Co}_{0.15} \mathrm{Al}_{0.05} \mathrm{O}_{2} / \mathrm{LiMn}_{1 / 3} \mathrm{Ni}_{1 / 3} \mathrm{Co}_{1 / 3} \mathrm{O}_{2}$ with core-shell structure synthesized by a coprecipitation and spray pyrolysis process. Funct. Mater. Lett. 11:1850083. doi: 10.1142/S1793604718500832

Qu, X., Yu, Z., Ruan, D., Dou, A., Su, M., Zhou, Y., et al. (2020). Enhanced electrochemical performance of ni-rich cathode materials with $\mathrm{Li}_{1.3} \mathrm{Al}_{0.3} \mathrm{Ti}_{1.7}\left(\mathrm{PO}_{4}\right)_{3}$ coating. Acs Sustain. Chem. Eng. 8, 5819-5830. doi: 10.1021/acssuschemeng.9b05539

Ryu, H.-H., Park, J.-K., Yoon, C. S., and Sun, K.-Y. (2018). Capacity fading of Ni-Rich Li $\mathrm{Ni}_{\mathrm{x}} \mathrm{Co}_{\mathrm{y}} \mathrm{Mn}_{1-\mathrm{x}-\mathrm{y}} \mathrm{O}_{2}(0.6 \leq \mathrm{x} \leq 0.95)$ cathodes for high-energydensity lithium-ion batteries: bulk or surface degradation? Chem. Mater. 30, 1155-1163. doi: 10.1021/acs.chemmater.7b05269

Sangwal, K. (2018). Nucleation and Crystal Growth: Metastability of Solutions and Melts. Hoboken, NJ: John Wiley \& Sons. 398-403 doi: 10.1002/9781119461616

Shi, X., Wang, C., Ma, X., and Sun, J. (2009). Synthesis and electrochemical properties of $\mathrm{LiNi}_{0.9} \mathrm{Co}_{0.1} \mathrm{O}_{2}$ cathode material for lithium secondary battery. Mater. Chem. Phys. 113, 780-783. doi: 10.1016/j.matchemphys.2008.08.015

Shi, X., Wang, C., Zhang, Y., Liu, Q., Li, H., Song, D., et al. (2014). Structure and electrochemical behaviors of spherical $\mathrm{Li}_{1+\mathrm{x}} \mathrm{Ni}_{0.5} \mathrm{Mn}_{0.5} \mathrm{O}_{2+\delta}$ synthesized by rheological phase reaction method. Electrochim. Acta 150, 89-98. doi: 10.1016/j.electacta.2014.10.151

Shoemaker, D. P., Hu, J.-H., Chung, D. Y., Halder, G. J., Chupas, P. J., Soderholm, L., et al. (2014). In situ studies of a platform for metastable inorganic crystal growth and materials discovery. Proc. Natl. Acad. Sci. U.S.A. 111, 10922-10927. doi: 10.1073/pnas.1406211111

Su, Y., Chen, G., Chen, L., Li, W., Zhang, Q., Yang, Z., et al. (2018). Exposing the $\{010\}$ planes by oriented self-assembly with nanosheets to improve the electrochemical performances of Ni-Rich $\mathrm{LiNi}_{0.8} \mathrm{Co}_{0.1} \mathrm{Mn}_{0.1} \mathrm{O}_{2}$ microspheres. ACS Appl. Mater. Inter.10, 6407-6414. doi: 10.1021/acsami.7b18933

Su, Y., Gu, D., Shao, Y., Wang, X., and Pan, F. (2019). Improved electrochemical performance of $\mathrm{LiNi}_{0.5} \mathrm{Mn}_{0.3} \mathrm{Co}_{0.2} \mathrm{O}_{2}$ electrodes coated by atomic-layer-deposited $\mathrm{Ta}_{2} \mathrm{O}_{5}$. Funct. Mater. Lett. 12:18501035. doi: $10.1142 /$ S1793604718501035

Tang, H., Xi, M. Y., Huang, X. M., Feng, C., Zhang, Y., and Zhang, K. L. (2002). Rheological phase reaction synthesis of lithium intercalation materials for rechargeable battery. J. Mater. Sci. Lett. 21, 999-1001. doi: 10.1023/A:1016052405985

Teshima, K., Inagaki, H., Tanaka, S., Yubuta, K., Hozumi, M., Kohama, K., et al. (2011). Growth of well-developed $\mathrm{Li}_{4} \mathrm{Ti}_{5} \mathrm{O}_{12}$ crystals by the cooling of a sodium chloride flux. Crys. Growth Des. 11, 4401-4405. doi: 10.1021/cg200578r

Vasquez, G., Huq, A., and Latturner, S., E. (2019). In situ neutron diffraction studies of the metal flux growth of $\mathrm{Ba} / \mathrm{Yb} / \mathrm{Mg} / \mathrm{Si}$ intermetallics. Inorg. Chem. 58, 8111-8119. doi: 10.1021/acs.inorgchem.9b00857

Wang, D., Kou, R., Ren, Y., C.-,Sun, J., Zhao, H., M.-,Zhang, J., et al. (2017). Synthetic control of kinetic reaction pathway and cationic ordering in high-ni layered oxide cathodes. Adv. Mater. 29:201606715. doi: 10.1002/adma.201606715

Wang, J., Yox, P., and Kovnir, K. (2020a). Flux growth of phosphide and arsenide crystals. Front. Chem. 8:186. doi: 10.3389/fchem.2020.00186

Wang, J.-L., Liang-Jie, Y., Yi-Yong, Y., Ju-Tang, S., and Ke-Li, Z. (2003). Preparation by a rheological phase reaction method and thermal decomposition reaction mechanism of nickelous salicylate tetrahydrate. Wuhan Uni. J. Nat. Sci. 8, 853-856. doi: 10.1007/BF02900830

Wang, L., Wu, B., Mu, D., Liu, X., Peng, Y., Xu, H., Liu, Q., et al. (2016). Singlecrystal $\mathrm{LiNi}_{0.6} \mathrm{Co}_{0.2} \mathrm{Mn}_{0.2} \mathrm{O}_{2}$ as high performance cathode materials for Li-ion batteries. J. Alloy. Compd. 674, 360-367. doi: 10.1016/j.jallcom.2016.03.061

Wang, T., Ren, K., Xiao, W., Dong, W., Qiao, H., Duan, A., et al. (2020b). Tuning the $\mathrm{Li} / \mathrm{Ni}$ disorder of the NMC811 cathode by thermally driven competition between lattice ordering and structure decomposition. J. Phys. Chem. C 124, 5600-5607. doi: 10.1021/acs.jpcc.0c00720 
Wei, G.-Z., Lu, X., Ke, S.-F., Huang, L., Li, T.-J., Wang, X.-Z., et al. (2010). Crystal habit-tuned nanoplate material of $\mathrm{Li}\left[\mathrm{Li}_{1 / 3-2 \mathrm{x} / 3} \mathrm{Ni}_{\mathrm{x}} \mathrm{Mn}_{2 / 3-\mathrm{x} / 3}\right] \mathrm{O}_{2}$ for high-rate performance lithium-ion batteries. Adv. Mater. 22, 4364-4367. doi: $10.1002 /$ adma.201001578

Wu, F., Maier, J., and Yu, Y. (2020). Guidelines and trends for next-generation rechargeable lithium and lithium-ion batteries. Chem. Soc. Rev. 49, 1569-1614. doi: 10.1039/C7CS00863E

Xiang, W., W.-,Liu, Y., Zhang, J., Wang, S., T.-,Zhang, T., Yin, K., et al. (2019). Controlled synthesis of nickel-rich layered oxide cathodes with preferentially exposed $\{010\}$ active facets for high rate and long cycling stable lithium-ion batteries. J. Alloy. Compd. 775, 72-80. doi: 10.1016/j.jallcom.2018.10.057

Xiao, H., Zhan, H., and Zhou, Y. H. (2004). Synthesis of layered-structure $\mathrm{LiMn}_{1-\mathrm{x}} \mathrm{Cr}_{\mathrm{x}} \mathrm{O}_{2}$ for lithium-ion batteries by the rheological phase method. Mater. Lett. 58, 1620-1624. doi: 10.1016/j.matlet.2003.09.050

Xu, G.-L., Liu, Q., Lau, K. K. S., Liu, Y., Liu, X., Gao, H., et al. (2019). Building ultraconformal protective layers on both secondary and primary particles of layered lithium transition metal oxide cathodes. Nat. Energy 4, 484-494. doi: 10.1038/s41560-019-0387-1

Yu, R., Zhang, X., Liu, T., Xu, X., Huang, Y., Wang, G., et al. (2017). Hierarchically structured lithium-rich layered oxide with exposed active $\{010\}$ planes as highrate-capability cathode for lithium-ion batteries. Acs Sustain. Chem. Eng. 5, 8970-8981. doi: 10.1021/acssuschemeng.7b01773

Zhang, S. S. (2020). Problems and their origins of Ni-rich layered oxide cathode materials. Energy Storage Mater. 24, 247-254. doi: 10.1016/j.ensm.2019.08.013

Zhao, J., Zhang, W., Huq, A., Misture, S., T., Zhang, B., et al. (2017). In situ probing and synthetic control of cationic ordering in ni-rich layered oxide cathodes. Adv. Energy Mater. 7:1601266. doi: 10.1002/aenm.2016 01266

Zhao, Y., Du, R., and Li, Y. (2011). Rheological phase synthesis and symbolization of single crystals of $\mathrm{Cu}\left(\mathrm{C}_{6} \mathrm{H}_{5} \mathrm{CO}_{2}\right)_{3} \mathrm{H}$ coordination compound. Appl. Chem. Ind. 40, 84-87. doi: 10.16581/j.cnki.issn1671-3206.2011. 01.029
Zheng, S. Q., Dou, A., C., Su, M., R., Liu, Y., et al. (2020). Influence of $\mathrm{Nb}$ doping on electrochemical performance of nanostructured cation disordered $\mathrm{Li}_{1+\mathrm{x} / 100} \mathrm{Ni}_{1 / 2-\mathrm{x} / 100} \mathrm{Ti}_{1 / 2-\mathrm{x} / 100} \mathrm{Nb}_{\mathrm{x} / 100} \mathrm{O}_{2}$ composites cathode for li-ion batteries. J. Nanosci. Nanotech. 20, 452-459. doi: 10.1166/jnn.2020.16884

Zhong, Z., Chen, L., Huang, S., Shang, W., Kong, L., Sun, M., et al. (2020). Single-crystal $\mathrm{LiNi}_{0.5} \mathrm{Co}_{0.2} \mathrm{Mn}_{0.3} \mathrm{O}_{2}$ : a high thermal and cycling stable cathodes for lithium-ion batteries. J. Mater. Sci. 55, 2913-2922. doi: 10.1007/s10853-019-04133-Z

Zhou, H., Xin, F., Pei, B., and Whittingham, M. S. (2019). What limits the capacity of layered oxide cathodes in lithium batteries? Acs Energy Lett. 4, 1902-1906. doi: 10.1021/acsenergylett.9b01236

Zhou, S., Mei, T., Wang, X., and Qian, Y. (2018). Crystal structural design of exposed planes: express channels, high-rate capability cathodes for lithium-ion batteries. Nanoscale 10, 17435-17455. doi: 10.1039/C8NR04842H

Zhu, J., Thinh, V., Li, D., Lu, R., Kinsinger, N., M., et al. (2012). Crystal growth of $\mathrm{Li} \mathrm{Ni}_{1 / 3} \mathrm{Co}_{1 / 3} \mathrm{Mn}_{1 / 3} \mathrm{O}_{2}$ as a cathode material for high-performance lithium ion batteries. Crys. Growth Des. 12, 1118-1123. doi: 10.1021/cg200565n

Zou, P., Lin, Z., Fan, M., Wang, F., Liu, Y., and Xiong, X. (2020). Facile and efficient fabrication of $\mathrm{Li}_{3} \mathrm{PO}_{4}$-coated $\mathrm{Ni}$-rich cathode for high-performance lithiumion battery. Appl. Surf. Sci. 504:144506. doi: 10.1016/j.apsusc.2019.144506

Conflict of Interest: The authors declare that the research was conducted in the absence of any commercial or financial relationships that could be construed as a potential conflict of interest.

Copyright (c) 2020 Wang, Ren, He, Dong, Xiao, Pan, Yang, Yang, Liu, Cao, Ma and Wang. This is an open-access article distributed under the terms of the Creative Commons Attribution License (CC BY). The use, distribution or reproduction in other forums is permitted, provided the original author(s) and the copyright owner(s) are credited and that the original publication in this journal is cited, in accordance with accepted academic practice. No use, distribution or reproduction is permitted which does not comply with these terms. 